\title{
Genotypic screening of wheat and their physiological responses under lead toxicity
}

\author{
Md Mostafizur Rahman ${ }^{1}$, Md Ruhul Amin Mintu ${ }^{1}$, Ahmad Humayan Kabir ${ }^{1 *}$ \& Md Firoz Alam ${ }^{1,2}$ \\ ${ }^{1}$ Department of Botany, University of Rajshahi, Rajshahi 6205, Bangladesh \\ ${ }^{2}$ Institute of Biological Sciences, University of Rajshahi, Rajshahi 6205, Bangladesh \\ *Email: ahmad.kabir@ru.ac.bd
}

\section{ARTICLE HISTORY}

Received: 12 January 2021

Accepted: 02 May 2021

Available online: 01 July 2021

KEYWORDS

Wheat

Lead $(\mathrm{Pb})$ stress

Hydroponic culture

$\mathrm{Pb}$ tolerance

Screening

\begin{abstract}
Lead $(\mathrm{Pb})$ is the second most harmful heavy metal contaminant in the environment and toxic for plant growth and development. Therefore, the identification and selection of plant genotypes tolerant to $\mathrm{Pb}$ stress are of great significance. In this study, twenty-six wheat lines (Triticum aestivum) were screened for $\mathrm{Pb}$ tolerance based on their morpho-physiological variations at the seedling stage with a rapid hydroponic technique using lead nitrate $\left(\mathrm{Pb}\left(\mathrm{NO}_{3}\right)_{2}\right)$ at two concentrations $(500 \mu \mathrm{M}$ and $1 \mathrm{mM})$ along with control. Wheat genotypes showed distinct variations in plant height, plant biomass and chlorophyll concentration in response to different concentrations of $\mathrm{Pb}$. Considering all parameters, Akbar was found most tolerant ( $\mathrm{T}$ ) with minimum RS (2.97) to Pb stress, followed by BARI Gom-31 (3.45), Barkat (3.54) and Sufi (3.65), while BARI Gom-26 (10.14) was most sensitive (S) followed by Khude Gom (9.69), BARI Gom-30 (8.79), LaIGom (8.76) and BARI Gom-32 respectively. More scores were seen in the remaining genotypes and were graded as moderately tolerant/resistant (MT) to $\mathrm{Pb}$ stress. Results showed that the resistant line had less damage to root and shoot characteristics along with chlorophyll score, thereby providing a hint about the $\mathrm{Pb}$ tolerance capacity of wheat genotypes at the seedling stage. Furthermore, findings indicate that $\mathrm{Pb}$ susceptibility in wheat is predominantly associated with a decrease in the $\mathrm{Pb}$ components of the root and shoot. We suggest Akbar as an elite genotype to cultivate or use in downstream studies on the basis of our findings to ensure an improved crop production relative to other varieties evaluated. These findings provide the necessary background for $\mathrm{Pb}$ cleansing and $\mathrm{Pb}$-free wheat development for environment and health safety.
\end{abstract}

\section{Introduction}

Wheat is the second most important crop after rice as it fulfils the protein and caloric requirement of the world's one-third population (1). According to FAO estimate, world would require around 840 million tonnes of wheat by 2050 from its current production level of 642 million tonnes. Given the growing demand of wheat for human consumption, it is estimated to grow at $1.6 \%$ per year by 2020 (2). This target will be achieved only if global wheat production is increased by $2.5 \%$ per annum (3). Though, various abiotic stresses are responsible for poor wheat growth (up to $50 \%$ ), such as drought, saline, poor soil fertility and heavy metals $(4,5)$. Under stress conditions, morphological and physiological characteristics are affected (6).

The term "heavy metals" refers to the group of metals and metalloids of relatively high atomic mass $\left(>4.5 \mathrm{~g} / \mathrm{cm}^{3}\right)$ that can cause toxicity problems (7). Pollution of air, soil and water resources with heavy metals is a global environmental issue $(8,9)$ because their contamination is harmful to humans, wildlife and agriculture (10). In addition, rapid industrialization and urbanization have caused pollution of the environment by heavy metals and their rates of mobilization and transport in the environment have greatly accelerated since the 1940 s $(11,12)$.

Contrasted to other heavy metals, lead $(\mathrm{Pb})$ is the second most harmful pollutant after arsenic and listed as "the chemical of great concern" according to the new European REACH (Registration, Evaluation, Authorization and Restriction of Chemicals) regulations (13) and its concentration in agricultural soil has rapidly increased and persists longer due to various anthropogenic inputs (14). Lead is not a bioessential ingredient, but it is readily consumed and stored in plants and not only effects development and production, it also reaches the food chain, creating human and animal health hazards (15-17). Ultrastructures of organs, tissues and subcellular components such as chloroplast, mitochondria,

C Rahman et al (2021). This is an open-access article distributed under the terms of the Creative Commons Attribution License, which permits unrestricted use, distribution and reproduction in any medium, provided the original author and source are credited (https://creativecommons.org/licenses/by/4.0/). 
nucleus, cell wall and plant cell membrane can also be impaired. In addition, this disruption may result in the loss of organelle function and may potentially affect the normal physiological functions of plants, including photosynthesis, respiration, protein synthesis and division of cells $(18,19)$. Lead may appear in soil as a free metal ion, complexed with inorganic constituents $\left(\mathrm{HCO}_{3}{ }^{-}, \mathrm{CO}_{3}{ }^{2-}, \mathrm{SO}_{4}{ }^{2-}\right.$, and $\left.\mathrm{Cl}^{-}\right)$, or as organic ligands (amino acids, fulvic acids, and humic acids); lead may alternatively be adsorbed on the surfaces of particles (Fe-oxides, biological material, organic matter and clay particles) (20-23).

Techniques for heavy metal restoration are classified as biological (biodegradation by living organisms), chemical (chelators, chemical immobilization, oxidation) and physical (electrokinetic remediation, incineration technologies, soil washing, stabilization/solidification, thermal desorption), which are costly, timeconsuming and environmentally hazardous (24). Thus, their removal/immobilization requires successful cleanup to mitigate or eradicate toxicity (25). Plants were suggested as a low-cost, sustainable and ecologically sound solution for the remediation of heavy metal-contaminated land (26), especially by phytoextraction (27). Different processes (physical, chemical and biological) are developed to reduce total $\mathrm{Pb}$ concentration and bioavailability to mitigate $\mathrm{Pb}$ accumulation in the food chain $(28,29)$. Lead $(\mathrm{Pb})$ uptake is usually limited to roots, with only slight translocation to the shoots (30-32). Plants also respond to harmful effects of lead in a variety of ways, such as selective metal uptake, metal binding to the root surface, cell wall binding and antioxidants induction: non-protein thiol (NP-SH), cysteine, glutathione, ascorbic acid, proline and antioxidant enzymes, such as superoxide dismutase (SOD), ascorbate peroxidase (APX), guaiacol peroxidase (GPX), catalase (CAT) and glutathione reductase (GR). These responses vary with plant species, metal content and exposure conditions (13).

Hydroponic methods are effective in the rapid screening for heavy metal tolerance and accumulation in plants and have been widely used in evaluating the phytoremediation potential $(33,34)$. The relative success of the hydroponically analyzed species is thus broadly comparable to that studied in the field (35). Wheat plants were chosen for this study because of their agricultural importance as a source of food whereas, 26 wheat genotypes were studied for their $\mathrm{Pb}$ tolerance based on morphological parameters as well as photosynthetic pigments content under hydroponics conditions. Thus, the present study was aimed at screening different wheat genotypes tolerant to $\mathrm{Pb}$ stress. A further purpose of this study was to establish the hydroponic method for screening wheat plants under $\mathrm{Pb}$ stress.

\section{Materials and Methods}

\section{Plant materials}

Twenty-six existing wheat genotypes in Bangladesh viz Kheri, Kalyansona, Sonora-64, Sonalika, Pavon-76,
Balaka, Kanchan, Akbar, Barkat, Sourav (BARI Gom19), Gourab (BARI Gom-20), Shatabdi (BARI Gom-21), Sufi (BARI Gom-22), Bijoy (BARI Gom-23), Prodip (BARI Gom-24), BARI Gom-25, BARI Gom-26, BARI Gom-27, BARI Gom-28, BARI Gom-29, BARI Gom-30, BARI Gom-31, BARI Gom-32, BARI Gom-33, LalGom (Red colors) and KhudeGom (small size) were used in this study. Initially, 24 genotypes of wheat seeds were collected from Bangladesh Regional Wheat Research Center, Rajshahi, Bangladesh, while LalGom from Rajshahi and KhudeGom from Meherpur, Bangladesh. All these wheat genotypes were identified by the Bangladesh Wheat and Maize Research Institute and their taxonomic details are available online (http://www.bwmri.gov.bd/).

\section{Germination and growth conditions for hydroponic culture}

Until germination, seeds were sterilized for 15 seconds in $70 \%$ ethanol and rinsed several times with purified water, then germinated for 3-4 days in Petri dishes containing two sheets of moist tissue paper in the dark at $25{ }^{\circ} \mathrm{C}$. After germination, seedlings of uniform size were transferred to black plastic pots (volume $600 \mathrm{ml}$ ) filled with half-strength Hoagland nutrition solution (36) with the following nutrient concentrations $(\mu \mathrm{M}): \mathrm{KNO}_{3}(16000), \mathrm{Ca}\left(\mathrm{NO}_{3}\right)_{2} \cdot 4 \mathrm{H}_{2} \mathrm{O}$ (6000), $\mathrm{NH}_{4} \mathrm{H}_{2} \mathrm{PO}_{4}$ (4000), $\mathrm{MgSO}_{4} .7 \mathrm{H}_{2} \mathrm{O}$ (2000), $\mathrm{KCl}(50)$, $\mathrm{H}_{3} \mathrm{BO}_{3}(25)$, Fe-EDTA (25), $\mathrm{MnSO}_{4} .4 \mathrm{H}_{2} \mathrm{O}$ (2), $\mathrm{Na}_{2} \mathrm{MoO}_{4} .2 \mathrm{H}_{2} \mathrm{O}(0.5), \mathrm{CuSO}_{4} .5 \mathrm{H}_{2} \mathrm{O}(0.5)$ at $25^{\circ} \mathrm{C}$ for 7 days. Under $10 \mathrm{hr}$ of light and $14 \mathrm{hr}$ of darkness (550$560 \mu \mathrm{mol} \mathrm{s}{ }^{-1}$ per $\mu \mathrm{A}$ ), the environment was strictly controlled. By using $\mathrm{NaOH}$ or $\mathrm{HCl}$, the $\mathrm{pH}$ was set to 6 . For $\mathrm{Pb}$ treatment, the culture solution in the hydroponic system was supplemented with two concentrations of $\mathrm{Pb}\left(\mathrm{NO}_{3}\right)_{2}(500 \mu \mathrm{M}$ and $1 \mathrm{mM})$ for seven days (37). Unstressed control plants were grown simultaneously and harvested. The treatments were arranged with three replicates in a completely randomized design.

\section{Measurement of morphological characters}

Plants were carefully taken from the pot after 7 days of treatment and washed twice with purified water to extract excess nutrient and then dried easily with tissue papers and then recorded the different morphological parameters such as root length (RL) plant $^{-1}$, shoot height (SH) plant $^{-1}$, root dry weight (RDW) plant ${ }^{-1}$ and shoot dry weight (SDW) plant ${ }^{-1}$. After incubation in an oven at $80^{\circ} \mathrm{C}$ until constant weight was achieved, shot and root dry weights were determined (37).

\section{Determination of chlorophyll score}

A portable chlorophyll meter or SPAD meter (atLEAF CHL STD, Wilmington, Delaware, USA) was used to take chlorophyll contents or SPAD values from the uppermost fully expanded leaves on each plant at $7^{\text {th }}$ day of treatments. A total of three plants were measured in every plot and took average as the mean SPAD value of the leaf (38).

\section{Statistical analysis}

The experiment was conducted with three replicates in a completely randomized design. Variations within the $\mathrm{Pb}$ concentrations and among the genotypes were 
checked using the computer package IBM SPSS Statistics 20 and MSTAT-C2.10 software and graphs were done using GraphPad Prism 6 software, respectively. Finally, the mean of the different parameters was compared by Duncan's Multiple Range Test (DMRT). Statistical significance was identified at $P \leq 0.05$. Means \pm standard deviations (SD) of three replicates for each treatment (Table 1 and Fig.1). For screening of $\mathrm{Pb}$ tolerant genotypes, a rank-sum (RS) was calculated by the following relationship (39): Rank sum (RS) = Rank mean $(\bar{R})+$ Standard deviation of rank (SDR).

\section{Results}

The findings of the variance analysis for the characters: root length, shoot height, root dry weight, shoot dry weight, as well as chlorophyll content was presented in Table 1 . The mean squares for the $\mathrm{Pb}$ treatment levels, genotypes and interaction between the treatments and genotypes $(\mathrm{AxB})$ were highly significant $(p<0.01)$ for all the characters suggesting the presence of considerable variations among the $\mathrm{Pb}$ treatments as well as genotypes.

\section{Root length and shoot height}

In Sonora-64, Sourav, LalGom, BARI Gom-26 and BARI Gom-27, root lengths were substantially reduced compared to controls for all $\mathrm{Pb}$ concentrations (Table 2). In addition, when treated with plants with $500 \mu \mathrm{M} \mathrm{Pb}$, Kheri, Kalyansona, Sonalika, Pavon-76, Gourab, Shatabdi, Bijoy, Prodip, BARI Gom-29, BARI Gom-31 and Balaka showed no started from $500 \mu \mathrm{M} \mathrm{Pb}$ in comparison with nontreated controls. Interestingly, no significant changes in shoot heights were observed in Sonalika, Kanchan, Sourav, Gourab, Shatabdi, Sufi, Bijoy, BARI Gom-25, LalGom, Akbar, Barkat, BARI Gom-27 and BARI Gom28 subjected to all levels of $\mathrm{Pb}$ compared with controls (Table 3).

\section{Root dry weight and shoot dry weight}

Compared to control, root dry weights were greatly reduced in Sourav and BARI Gom-26 supplemented with both $\mathrm{Pb}$ concentrations (Table 4). Furthermore, when plants were treated with $500 \mu \mathrm{M} \mathrm{Pb}$, Kheri, Sonora-64, Pavon-76, Gourab, Bijoy and BARI Gom-29 displayed no substantial reduction in root dry weights but a major reduction in $1 \mathrm{mM} \mathrm{Pb}$ compared to controls (Table 4). However, BARI Gom-32, BARI Gom-33, LalGom, Balaka, BARI Gom-28 and BARI Gom-30 showed a significant decrease in root dry weight started from $500 \mu \mathrm{M} \mathrm{Pb}$ in comparison with non-treated controls. Interestingly, no significant changes in root dry weights were observed in Kalyansona, Sonalika, Kanchan, Shatabdi, Sufi, Prodip, BARI Gom-25, BARI Gom-31, KhudeGom, Akbar, Barkat and BARI Gom-27 subjected to all levels of $\mathrm{Pb}$ compared with controls (Table 4).

Shoot dry weights were significantly decreased only in BARI Gom-26 supplemented with all concentrations of $\mathrm{Pb}$ compared with control (Table 5). However, when plants were treated with $500 \mu \mathrm{M}$ $\mathrm{Pb}$, Kheri, Kalyansona, BARI Gom-29 and KhudeGom displayed no substantial reduction in shoot dry weights but a major reduction in $1 \mathrm{mM} \mathrm{Pb}$ compared

Table 1. Mean squares of 26 wheat genotypes for various characters recorded in laboratory conditions under control and different Pb stress levels.

\begin{tabular}{|c|c|c|c|c|c|}
\hline \multirow[b]{2}{*}{ Characters } & \multicolumn{5}{|c|}{ Sources of variation } \\
\hline & $\begin{array}{c}\text { Factor A } \\
\text { (Pb treatments) } \\
\text { df }=2\end{array}$ & $\begin{array}{c}\text { Factor B } \\
\text { (Genotypes) } \\
\text { df }=25\end{array}$ & $\begin{array}{c}\text { A X B } \\
\text { (Interaction) } \\
\text { df }=50\end{array}$ & $\begin{array}{c}\text { Error } \\
\text { df }=156\end{array}$ & $\begin{array}{l}\text { Co-efficient of } \\
\text { variation (CV) }\end{array}$ \\
\hline Root length & 452.607 & 72.432 & 34.509 & 2.061 & $12.45 \%$ \\
\hline Shoot height & 77.039 & 33.905 & 7.781 & 1.955 & $7.56 \%$ \\
\hline Root dry weight & 0.000 & 0.000 & 0.000 & 0.000 & $18.39 \%$ \\
\hline Shoot dry weight & 0.000 & 0.000 & 0.000 & 0.000 & $15.21 \%$ \\
\hline Chlorophyll content/SPAD & 56.602 & 82.338 & 23.199 & 13.242 & $8.30 \%$ \\
\hline
\end{tabular}

substantial reduction in root lengths, but a major reduction in $1 \mathrm{mM} \mathrm{Pb}$ compared to controls. However, BARI Gom-32, BARI Gom-33, KhudeGom, BARI Gom-28 and BARI Gom-30 showed a significant reduction in root lengths started from $500 \mu \mathrm{M} \mathrm{Pb}$ in comparison with non-treated controls. Interestingly, no significant changes were found in the root lengths of Kanchan, Sufi, BARI Gom-25, Akbar and Barkat at all levels of $\mathrm{Pb}$ compared to control levels (Table 2).

Shoot height decreased dramatically only in BARI Gom-26, complemented with all $\mathrm{Pb}$ concentrations relative to control (Table 3). However, when plants were treated with $500 \mu \mathrm{M} \mathrm{Pb}$, Kheri, Kalyansona, Sonora-64, Pavon-76, Prodip, BARI Gom29, BARI Gom-31, KhudeGom and Balaka displayed no substantial reduction in shoot heights, but a major reduction in $1 \mathrm{mM} \mathrm{Pb}$ compared to controls. Further, BARI Gom-32, BARI Gom-33 and BARI Gom-30 showed a significant reduction in shoot heights to the controls (Table 5). Further, BARI Gom-32, BARI Gom-33, Balaka and BARI Gom-30 showed a significant decrease in shoot dry weights concentration started from $500 \mu \mathrm{M} \mathrm{Pb}$ in comparison with non-treated controls. Interestingly, no significant changes in shoot dry weights were observed in Sonora-64, Sonalika, Pavon-76, Kanchan, Sourav, Gourab, Shatabdi, Sufi, Bijoy, Prodip, BARI Gom-25, BARI Gom-31, LalGom, Akbar, Barkat, BARI Gom-27 and BARI Gom-28 subjected to all levels of $\mathrm{Pb}$ compared with controls (Table 5).

\section{Chlorophyll concentrations in leaves}

Total chlorophyll concentrations were measured in the leaves of wheat genotypes cultivated in the absence and presence $\mathrm{Pb}$ (Table 6). When plants were treated with $500 \mu \mathrm{M} \mathrm{Pb}$, Kheri, Sonora-64, BARI Gom31, BARI Gom-32, KhudeGom and BARI Gom-30 displayed no substantial reduction in chlorophyll score but a major reduction in $1 \mathrm{mM} \mathrm{Pb}$ compared to 
controls (Table 6). However, chlorophyll score was significantly decreased only in Barkat started from $500 \mu \mathrm{M} \mathrm{Pb}$. Interestingly, no significant differences in chlorophyll scores were observed in Kalyansona, Sonalika, Pavon-76, Kanchan, Sourav, Gourab, Shatabdi, Sufi, Bijoy, Prodip, BARI Gom-25, BARI Gom-29, BARI Gom-33, LalGom, Balaka, Akbar, BARI
Gom-26, BARI Gom-27 and BARI Gom-28 subjected to all levels of $\mathrm{Pb}$ compared with controls (Table 6).

\section{Ranking of the genotypes}

In order to find out the performance of genotypes for $\mathrm{Pb}$ tolerance, mean rank, the standard deviation of ranks and rank-sum were calculated, ranked and

Table 2. Measurement of root length (cm) in various genotypes of wheat cultivated without (control) and with varying levels of $\mathrm{Pb}(500 \mu \mathrm{M}$ and $\left.1 \mathrm{mM} \mathrm{Pb}\left(\mathrm{NO}_{3}\right)_{2}\right)$. Values are the means \pm SD of three replicates $(n=3)$. In accordance with the DMRT, various superscripted letters $(\mathrm{a}-\mathrm{c})$ within the row suggest statistically significant variations between the treatments $(\mathrm{P}<0.05)$. Data were taken at $7^{\text {th }}$ day of treatments.

\begin{tabular}{|c|c|c|c|}
\hline \multirow{2}{*}{ Genotypes } & \multicolumn{3}{|c|}{ Treatments } \\
\hline & Control & $500 \mu \mathrm{M}$ & $1 \mathrm{mM}$ \\
\hline Kheri & $13.1333 \pm 2.3072^{\mathrm{b}}$ & $10.9333 \pm 1.1930^{\mathrm{b}}$ & $3.9333 \pm 0.7371^{\mathrm{a}}$ \\
\hline Kalyansona & $13.8333 \pm 0.2887^{b}$ & $13.0333 \pm 1.3650^{\mathrm{b}}$ & $4.8000 \pm 0.5568^{\mathrm{a}}$ \\
\hline Sonora-64 & $16.6000 \pm 1.6643^{\mathrm{c}}$ & $11.2000 \pm 1.4731^{\mathrm{b}}$ & $4.0667 \pm 0.1155^{\mathrm{a}}$ \\
\hline Sonalika & $13.0000 \pm 3.7000^{\mathrm{b}}$ & $11.3000 \pm 2.4880^{\mathrm{ab}}$ & $6.7333 \pm 0.4619^{a}$ \\
\hline Pavon-76 & $16.1333 \pm 0.2082^{\mathrm{b}}$ & $17.3333 \pm 0.8145^{\mathrm{b}}$ & $4.2000 \pm 0.7000^{\mathrm{a}}$ \\
\hline Kanchan & $16.3333 \pm 1.0263^{\mathrm{a}}$ & $15.5667 \pm 2.0793^{\mathrm{a}}$ & $17.0333 \pm 1.0970^{\mathrm{a}}$ \\
\hline Sourav & $15.8000 \pm 1.4000^{c}$ & $13.2667 \pm 0.5686^{\mathrm{b}}$ & $7.9667 \pm 0.9866^{\mathrm{a}}$ \\
\hline Gourab & $14.8000 \pm 2.6287^{\mathrm{b}}$ & $13.1000 \pm 1.6000^{\mathrm{ab}}$ & $9.8667 \pm 0.8145^{a}$ \\
\hline Shatabdi & $14.1667 \pm 0.3786^{\mathrm{b}}$ & $13.8000 \pm 1.4177^{b}$ & $8.0000 \pm 1.4177^{a}$ \\
\hline Sufi & $17.6333 \pm 0.7572^{\mathrm{a}}$ & $17.1000 \pm 1.3077^{\mathrm{a}}$ & $17.6667 \pm 0.5860^{\mathrm{a}}$ \\
\hline Bijoy & $13.8333 \pm 1.6503^{b}$ & $13.5333 \pm 1.3614^{\mathrm{b}}$ & $9.6000 \pm 1.3229^{\mathrm{a}}$ \\
\hline Prodip & $14.9000 \pm 1.6523^{b}$ & $13.2000 \pm 0.9539^{\mathrm{ab}}$ & $12.2000 \pm 0.8185^{\mathrm{a}}$ \\
\hline BARI Gom-25 & $11.1667 \pm 0.1528^{\mathrm{a}}$ & $10.4667 \pm 3.5949^{\mathrm{a}}$ & $11.9667 \pm 1.3317^{\mathrm{a}}$ \\
\hline BARI Gom-29 & $19.4667 \pm 0.4163^{b}$ & $17.8333 \pm 1.1676^{\mathrm{b}}$ & $11.3000 \pm 2.0664^{\mathrm{a}}$ \\
\hline BARI Gom-31 & $16.9000 \pm 1.8358^{\mathrm{b}}$ & $16.7667 \pm 0.4163^{b}$ & $13.5333 \pm 0.1155^{\mathrm{a}}$ \\
\hline BARI Gom-32 & $15.8667 \pm 0.4726^{\mathrm{b}}$ & $5.5667 \pm 1.3503^{a}$ & $6.1333 \pm 0.4726^{\mathrm{a}}$ \\
\hline BARI Gom-33 & $16.7000 \pm 1.1533^{\mathrm{b}}$ & $6.5667 \pm 0.4041^{\mathrm{a}}$ & $6.1333 \pm 1.3650^{a}$ \\
\hline LalGom & $16.5667 \pm 1.6258^{c}$ & $6.0667 \pm 0.6658^{\mathrm{b}}$ & $2.5000 \pm 0.4583^{a}$ \\
\hline KhudeGom & $11.0667 \pm 1.2503^{\mathrm{b}}$ & $4.4333 \pm 1.1590^{\mathrm{a}}$ & $5.5333 \pm 1.8610^{a}$ \\
\hline Balaka & $15.5333 \pm 1.2662^{\mathrm{b}}$ & $14.5667 \pm 0.5508^{b}$ & $4.9667 \pm 0.5508^{\mathrm{a}}$ \\
\hline Akbar & $11.8667 \pm 0.8737^{\mathrm{a}}$ & $13.7000 \pm 1.4107^{\mathrm{a}}$ & $13.7000 \pm 1.8735^{a}$ \\
\hline Barkat & $16.2333 \pm 1.2503^{\mathrm{a}}$ & $16.5667 \pm 0.4041^{\mathrm{a}}$ & $16.8333 \pm 2.0648^{a}$ \\
\hline BARI Gom-26 & $10.3667 \pm 0.5859^{c}$ & $5.6000 \pm 0.9539^{\mathrm{b}}$ & $3.3000 \pm 0.6557^{a}$ \\
\hline BARI Gom-27 & $14.2000 \pm 0.8185^{c}$ & $10.2667 \pm 1.6623^{\mathrm{b}}$ & $7.6000 \pm 1.3115^{\mathrm{a}}$ \\
\hline BARI Gom-28 & $11.4333 \pm 1.5948^{\mathrm{b}}$ & $6.8333 \pm 0.3055^{\mathrm{a}}$ & $6.0667 \pm 0.5132^{\mathrm{a}}$ \\
\hline BARI Gom-30 & $13.7667 \pm 1.5631^{\mathrm{b}}$ & $3.7000 \pm 0.5000^{\mathrm{a}}$ & $5.0333 \pm 0.3215^{\mathrm{a}}$ \\
\hline
\end{tabular}

Table 3. Measurement of shoot height $(\mathrm{cm})$ in various genotypes of wheat cultivated without (control) and with varying levels of $\mathrm{Pb}$ (500 $\mu \mathrm{M}$ and $\left.1 \mathrm{mM} \mathrm{Pb}\left(\mathrm{NO}_{3}\right)_{2}\right)$. Values are the means $\pm \mathrm{SD}$ of three replicates $(n=3)$. In accordance with the DMRT, various superscripted letters (a-c) within the row suggest statistically significant variations between the treatments $(P<0.05)$. Data were taken at $7^{\text {th }}$ day of treatments.

\begin{tabular}{|c|c|c|c|}
\hline \multirow{2}{*}{ Genotypes } & \multicolumn{3}{|c|}{ Treatments } \\
\hline & Control & $500 \mu \mathrm{M}$ & $1 \mathrm{mM}$ \\
\hline Kheri & $19.2000 \pm 4.2320^{\mathrm{b}}$ & $15.3000 \pm 3.1953^{\mathrm{ab}}$ & $11.4333 \pm 0.8963^{a}$ \\
\hline Kalyansona & $19.0667 \pm 0.6351^{\mathrm{b}}$ & $17.6000 \pm 0.7810^{\mathrm{b}}$ & $14.0333 \pm 1.7502^{\mathrm{a}}$ \\
\hline Sonora-64 & $19.9667 \pm 1.1015^{b}$ & $17.5000 \pm 0.4583^{\mathrm{ab}}$ & $16.0333 \pm 2.6633^{a}$ \\
\hline Sonalika & $17.5000 \pm 0.5292^{\mathrm{a}}$ & $18.0333 \pm 1.5822^{\mathrm{a}}$ & $17.3667 \pm 1.7616^{a}$ \\
\hline Pavon-76 & $18.2000 \pm 0.3606^{\mathrm{b}}$ & $18.1667 \pm 0.6809^{b}$ & $16.6667 \pm 0.8737^{a}$ \\
\hline Kanchan & $17.7000 \pm 0.3606^{\mathrm{a}}$ & $18.6333 \pm 1.5503^{\mathrm{a}}$ & $17.6000 \pm 0.7937^{a}$ \\
\hline Sourav & $19.1000 \pm 0.5000^{\mathrm{a}}$ & $18.7333 \pm 0.8083^{\mathrm{a}}$ & $18.5333 \pm 0.5508^{a}$ \\
\hline Gourab & $20.3667 \pm 1.0066^{\mathrm{a}}$ & $18.8000 \pm 2.0881^{\mathrm{a}}$ & $18.4667 \pm 0.6658^{a}$ \\
\hline Shatabdi & $20.0000 \pm 1.4107^{a}$ & $20.0333 \pm 0.7024^{\mathrm{a}}$ & $20.1667 \pm 0.8327^{a}$ \\
\hline Sufi & $21.9000 \pm 2.8054^{\mathrm{a}}$ & $23.9333 \pm 0.5132^{\mathrm{a}}$ & $23.5000 \pm 0.3606^{a}$ \\
\hline Bijoy & $19.4000 \pm 0.3464^{\mathrm{a}}$ & $19.5000 \pm 1.3077^{\mathrm{a}}$ & $18.7000 \pm 1.5524^{\mathrm{a}}$ \\
\hline Prodip & $21.0333 \pm 0.4619^{b}$ & $21.4333 \pm 0.4163^{b}$ & $19.2333 \pm 0.9019^{a}$ \\
\hline BARI Gom-25 & $20.1667 \pm 1.2014^{\mathrm{a}}$ & $20.5000 \pm 1.3748^{\mathrm{a}}$ & $19.0667 \pm 0.5132^{a}$ \\
\hline BARI Gom-29 & $21.1000 \pm 1.3528^{\mathrm{b}}$ & $20.1000 \pm 0.4000^{\mathrm{b}}$ & $17.8000 \pm 0.9849^{a}$ \\
\hline BARI Gom-31 & $21.0667 \pm 0.2309^{b}$ & $20.8000 \pm 1.0583^{\mathrm{ab}}$ & $19.3333 \pm 0.6807^{a}$ \\
\hline BARI Gom-32 & $18.1667 \pm 2.0207^{b}$ & $14.5333 \pm 0.4041^{\mathrm{a}}$ & $14.5333 \pm 0.8963^{a}$ \\
\hline$\overline{\text { BARI Gom-33 }}$ & $21.4333 \pm 0.0551^{b}$ & $17.3333 \pm 0.5033^{\mathrm{a}}$ & $15.6333 \pm 2.2368^{a}$ \\
\hline LalGom & $18.2000 \pm 0.4000^{\mathrm{a}}$ & $13.6000 \pm 1.4000^{\mathrm{a}}$ & $13.3333 \pm 3.8175^{a}$ \\
\hline KhudeGom & $16.5333 \pm 0.1155^{b}$ & $15.5333 \pm 0.1528^{b}$ & $12.5000 \pm 1.4000^{\mathrm{a}}$ \\
\hline Balaka & $18.6667 \pm 1.1060^{b}$ & $19.8667 \pm 0.8083^{b}$ & $13.6333 \pm 0.3215^{a}$ \\
\hline Akbar & $21.2000 \pm 1.3747^{\mathrm{a}}$ & $20.9000 \pm 0.5000^{\mathrm{a}}$ & $20.1667 \pm 1.4224^{a}$ \\
\hline Barkat & $19.5000 \pm 0.9539^{a}$ & $18.8667 \pm 0.4041^{\mathrm{a}}$ & $19.1333 \pm 1.1060^{a}$ \\
\hline BARI Gom-26 & $19.2667 \pm 1.1719^{c}$ & $14.0667 \pm 0.6351^{\mathrm{b}}$ & $11.2000 \pm 0.9644^{\mathrm{a}}$ \\
\hline BARI Gom-27 & $20.0000 \pm 1.5588^{\mathrm{a}}$ & $18.0667 \pm 1.4012^{\mathrm{a}}$ & $16.5000 \pm 2.4637^{a}$ \\
\hline BARI Gom-28 & $16.4000 \pm 0.4000^{\mathrm{a}}$ & $17.0667 \pm 1.1015^{\mathrm{a}}$ & $17.7000 \pm 1.1358^{a}$ \\
\hline BARI Gom-30 & $21.2333 \pm 0.6658^{\mathrm{b}}$ & $16.0667 \pm 1.5275^{\mathrm{a}}$ & $16.3000 \pm 1.0149^{a}$ \\
\hline
\end{tabular}


Table 4. Measurement of root dry weight (gm) in various genotypes of wheat cultivated without (control) and with varying levels of $\mathrm{Pb}$ $\left(500 \mu \mathrm{M}\right.$ and $\left.1 \mathrm{mM} \mathrm{Pb}\left(\mathrm{NO}_{3}\right)_{2}\right)$. Values are the means $\pm \mathrm{SD}$ of three replicates $(\mathrm{n}=3)$. In accordance with the DMRT, various superscripted letters $(\mathrm{a}-\mathrm{c})$ within the row suggest statistically significant variations between the treatments $(\mathrm{P}<0.05)$. Data were taken at $7^{\text {th }}$ day of treatments.

\begin{tabular}{|c|c|c|c|}
\hline \multirow{2}{*}{ Genotypes } & \multicolumn{3}{|c|}{ Treatments } \\
\hline & Control & $500 \mu \mathrm{M}$ & $1 \mathrm{mM}$ \\
\hline Kheri & $0.0061 \pm 0.0011^{b}$ & $0.0047 \pm 0.0008^{\mathrm{ab}}$ & $0.0035 \pm 0.0015^{a}$ \\
\hline Kalyansona & $0.0066 \pm 0.0012^{a}$ & $0.0065 \pm 0.0007^{a}$ & $0.0052 \pm 0.0001^{\mathrm{a}}$ \\
\hline Sonora-64 & $0.0085 \pm 0.0007^{b}$ & $0.0073 \pm 0.0011^{\mathrm{b}}$ & $0.0034 \pm 0.0076^{\mathrm{a}}$ \\
\hline Sonalika & $0.0049 \pm 0.0005^{a}$ & $0.0048 \pm 0.0003^{a}$ & $0.0046 \pm 0.0002^{\mathrm{a}}$ \\
\hline Pavon-76 & $0.0066 \pm 0.0005^{b}$ & $0.0069 \pm 0.0005^{b}$ & $0.0043 \pm 0.0004^{\mathrm{a}}$ \\
\hline Kanchan & $0.0070 \pm 0.0014^{\mathrm{a}}$ & $0.0070 \pm 0.0015^{a}$ & $0.0074 \pm 0.0006^{\mathrm{a}}$ \\
\hline Sourav & $0.0113 \pm 0.0008^{c}$ & $0.0091 \pm 0.0007^{\mathrm{b}}$ & $0.0073 \pm 0.0008^{a}$ \\
\hline Gourab & $0.0085 \pm 0.0009^{b}$ & $0.0079 \pm 0.0008^{b}$ & $0.0044 \pm 0.0008^{a}$ \\
\hline Shatabdi & $0.0074 \pm 0.0010^{\mathrm{a}}$ & $0.0089 \pm 0.0019^{a}$ & $0.0063 \pm 0.0011^{a}$ \\
\hline Sufi & $0.0064 \pm 0.0006^{\mathrm{a}}$ & $0.0057 \pm 0.0009^{a}$ & $0.0058 \pm 0.0001^{\mathrm{a}}$ \\
\hline Bijoy & $0.0086 \pm 0.0013^{b}$ & $0.0078 \pm 0.0051^{\mathrm{ab}}$ & $0.0068 \pm 0.0002^{\mathrm{a}}$ \\
\hline Prodip & $0.0073 \pm 0.0012^{\mathrm{a}}$ & $0.0077 \pm 0.0017^{a}$ & $0.0077 \pm 0.0004^{a}$ \\
\hline BARI Gom-25 & $0.0095 \pm 0.0002^{\mathrm{a}}$ & $0.0097 \pm 0.0015^{a}$ & $0.0093 \pm 0.0005^{\mathrm{a}}$ \\
\hline BARI Gom-29 & $0.0087 \pm 0.0012^{b}$ & $0.0086 \pm 0.0006^{b}$ & $0.0047 \pm 0.0012^{\mathrm{a}}$ \\
\hline BARI Gom-31 & $0.0060 \pm 0.0010^{\mathrm{a}}$ & $0.0080 \pm 0.0026^{\mathrm{a}}$ & $0.0077 \pm 0.0031^{\mathrm{a}}$ \\
\hline BARI Gom-32 & $0.0062 \pm 0.0006^{b}$ & $0.0036 \pm 0.0012^{\mathrm{a}}$ & $0.0040 \pm 0.0004^{a}$ \\
\hline BARI Gom-33 & $0.0084 \pm 0.0008^{\mathrm{b}}$ & $0.0046 \pm 0.0001^{\mathrm{a}}$ & $0.0050 \pm 0.0009^{a}$ \\
\hline LalGom & $0.0057 \pm 0.0002^{\mathrm{b}}$ & $0.0043 \pm 0.0005^{a}$ & $0.0031 \pm 0.0009^{a}$ \\
\hline KhudeGom & $0.0032 \pm 0.0001^{\mathrm{a}}$ & $0.0022 \pm 0.0005^{a}$ & $0.0024 \pm 0.0008^{a}$ \\
\hline Balaka & $0.0128 \pm 0.0006^{b}$ & $0.0062 \pm 0.0005^{a}$ & $0.0054 \pm 0.0013^{a}$ \\
\hline Akbar & $0.0070 \pm 0.0005^{a}$ & $0.0072 \pm 0.0017^{a}$ & $0.0085 \pm 0.0013^{a}$ \\
\hline Barkat & $0.0080 \pm 0.0006^{\mathrm{a}}$ & $0.0070 \pm 0.0007^{a}$ & $0.0092 \pm 0.0025^{a}$ \\
\hline BARI Gom-26 & $0.0078 \pm 0.0008^{c}$ & $0.0042 \pm 0.0006^{\mathrm{b}}$ & $0.0029 \pm 0.0005^{\mathrm{a}}$ \\
\hline BARI Gom-27 & $0.0073 \pm 0.0002^{\mathrm{a}}$ & $0.0059 \pm 0.0006^{a}$ & $0.0055 \pm 0.0022^{\mathrm{a}}$ \\
\hline BARI Gom-28 & $0.0077 \pm 0.0085^{b}$ & $0.0063 \pm 0.0004^{\mathrm{a}}$ & $0.0055 \pm 0.0005^{a}$ \\
\hline BARI Gom-30 & $0.0077 \pm 0.0014^{b}$ & $0.0038 \pm 0.0026^{a}$ & $0.0039 \pm 0.0011^{\mathrm{a}}$ \\
\hline
\end{tabular}

Table 5. Measurement of shoot dry weight (gm) in various genotypes of wheat cultivated without (control) and with varying levels of $\mathrm{Pb}$ $\left(500 \mu \mathrm{M}\right.$ and $\left.1 \mathrm{mM} \mathrm{Pb}\left(\mathrm{NO}_{3}\right)_{2}\right)$. Values are the means \pm SD of three replicates $(\mathrm{n}=3)$. In accordance with the DMRT, various superscripted letters $(\mathrm{a}-\mathrm{c})$ within the row suggest statistically significant variations between the treatments $(\mathrm{P}<0.05)$. Data were taken at $7^{\text {th }}$ day of treatments.

\begin{tabular}{|c|c|c|c|}
\hline \multirow{2}{*}{ Genotypes } & \multicolumn{3}{|c|}{ Treatments } \\
\hline & Control & $500 \mu \mathrm{M}$ & $1 \mathrm{mM}$ \\
\hline Kheri & $0.0185 \pm 0.0017^{b}$ & $0.0162 \pm 0.0017^{\mathrm{ab}}$ & $0.0134 \pm 0.0011^{\mathrm{a}}$ \\
\hline Kalyansona & $0.0179 \pm 0.0016^{\mathrm{b}}$ & $0.0182 \pm 0.0013^{b}$ & $0.0133 \pm 0.0011^{\mathrm{a}}$ \\
\hline Sonora-64 & $0.0208 \pm 0.0040^{\mathrm{a}}$ & $0.0204 \pm 0.0041^{\mathrm{a}}$ & $0.0135 \pm 0.0052^{\mathrm{a}}$ \\
\hline Sonalika & $0.0147 \pm 0.0006^{\mathrm{a}}$ & $0.0149 \pm 0.0009^{\mathrm{a}}$ & $0.0152 \pm 0.0004^{\mathrm{a}}$ \\
\hline Pavon-76 & $0.0184 \pm 0.0021^{\mathrm{a}}$ & $0.0179 \pm 0.0016^{\mathrm{a}}$ & $0.0145 \pm 0.0023^{\mathrm{a}}$ \\
\hline Kanchan & $0.0210 \pm 0.0033^{\mathrm{a}}$ & $0.0203 \pm 0.0005^{\mathrm{a}}$ & $0.0181 \pm 0.0010^{\mathrm{a}}$ \\
\hline Sourav & $0.0222 \pm 0.0022^{\mathrm{a}}$ & $0.0226 \pm 0.0014^{\mathrm{a}}$ & $0.0216 \pm 0.0023^{\mathrm{a}}$ \\
\hline Gourab & $0.0165 \pm 0.0009^{\mathrm{a}}$ & $0.0154 \pm 0.0020^{\mathrm{a}}$ & $0.0139 \pm 0.0028^{a}$ \\
\hline Shatabdi & $0.0224 \pm 0.0032^{\mathrm{a}}$ & $0.0209 \pm 0.0021^{\mathrm{a}}$ & $0.0202 \pm 0.0012^{\mathrm{a}}$ \\
\hline $\begin{array}{l}\text { Sufi } \\
\end{array}$ & $0.0232 \pm 0.0016^{\mathrm{a}}$ & $0.0238 \pm 0.0031^{\mathrm{a}}$ & $0.0209 \pm 0.0016^{\mathrm{a}}$ \\
\hline Bijoy & $0.0248 \pm 0.0036^{\mathrm{a}}$ & $0.0240 \pm 0.0008^{\mathrm{a}}$ & $0.0239 \pm 0.0038^{a}$ \\
\hline Prodip & $0.0230 \pm 0.0008^{\mathrm{a}}$ & $0.0224 \pm 0.0030^{\mathrm{a}}$ & $0.0224 \pm 0.0026^{\mathrm{a}}$ \\
\hline BARI Gom-25 & $0.0234 \pm 0.0042^{\mathrm{a}}$ & $0.0263 \pm 0.0040^{\mathrm{a}}$ & $0.0252 \pm 0.0025^{\mathrm{a}}$ \\
\hline BARI Gom-29 & $0.0253 \pm 0.0035^{b}$ & $0.0220 \pm 0.0017^{b}$ & $0.0133 \pm 0.0015^{\mathrm{a}}$ \\
\hline BARI Gom-31 & $0.0200 \pm 0.0036^{\mathrm{a}}$ & $0.0187 \pm 0.0038^{\mathrm{a}}$ & $0.0150 \pm 0.0030^{\mathrm{a}}$ \\
\hline BARI Gom-32 & $0.0175 \pm 0.0013^{b}$ & $0.0128 \pm 0.0023^{\mathrm{a}}$ & $0.0133 \pm 0.0016^{\mathrm{a}}$ \\
\hline BARI Gom-33 & $0.0249 \pm 0.0018^{b}$ & $0.0179 \pm 0.0019^{\mathrm{a}}$ & $0.0160 \pm 0.0033^{\mathrm{a}}$ \\
\hline LalGom & $0.0186 \pm 0.0007^{\mathrm{a}}$ & $0.0137 \pm 0.0016^{\mathrm{a}}$ & $0.0137 \pm 0.0061^{\mathrm{a}}$ \\
\hline KhudeGom & $0.0089 \pm 0.0004^{\mathrm{b}}$ & $0.0090 \pm 0.0006^{\mathrm{b}}$ & $0.0062 \pm 0.0008^{\mathrm{a}}$ \\
\hline Balaka & $0.0248 \pm 0.0034^{\mathrm{b}}$ & $0.0193 \pm 0.0019^{\mathrm{a}}$ & $0.0151 \pm 0.0010^{\mathrm{a}}$ \\
\hline Akbar & $0.0211 \pm 0.0024^{\mathrm{a}}$ & $0.0245 \pm 0.0022^{\mathrm{a}}$ & $0.0208 \pm 0.0031^{\mathrm{a}}$ \\
\hline Barkat & $0.0235 \pm 0.0009^{\mathrm{a}}$ & $0.0181 \pm 0.0026^{\mathrm{a}}$ & $0.0214 \pm 0.0041^{\mathrm{a}}$ \\
\hline BARI Gom-26 & $0.0183 \pm 0.0017^{c}$ & $0.0145 \pm 0.0019^{b}$ & $0.0108 \pm 0.0013^{\mathrm{a}}$ \\
\hline BARI Gom-27 & $0.0178 \pm 0.0013^{\mathrm{a}}$ & $0.0183 \pm 0.0019^{\mathrm{a}}$ & $0.0172 \pm 0.0015^{\mathrm{a}}$ \\
\hline BARI Gom-28 & $0.0184 \pm 0.0034^{\mathrm{a}}$ & $0.0179 \pm 0.0011^{\mathrm{a}}$ & $0.0154 \pm 0.0010^{\mathrm{a}}$ \\
\hline BARI Gom-30 & $0.0215 \pm 0.0012^{\mathrm{b}}$ & $0.0147 \pm 0.0037^{\mathrm{a}}$ & $0.0147 \pm 0.0003^{\mathrm{a}}$ \\
\hline
\end{tabular}

presented in Table 7. Scoring and ranking were conducted on the basis of the overall genotype performances on the statistical relevance of $\mathrm{Pb}$ treatments and controls. The lowest ranked sum reveals the best performing and the greater ranked sum indicates sensitive genotypes in response to $\mathrm{Pb}$ stress.

In response to $\mathrm{Pb}$ stress, the lowest ranked sum displays the best performance and the higher ranked 
Table 6. Measurement of chlorophyll contents or SPAD values from the uppermost fully expanded leaves in different wheat genotypes grown without (control) and with different $\mathrm{Pb}$ concentrations $\left(500 \mu \mathrm{M}\right.$ and $\left.1 \mathrm{mM} \mathrm{Pb}\left(\mathrm{NO}_{3}\right)_{2}\right)$. Values are the means $\pm \mathrm{SD}$ of three replicates $(\mathrm{n}=3)$. In accordance with the DMRT, various superscripted letters $(\mathrm{a}-\mathrm{c})$ within the row suggest statistically significant variations between the treatments $(\mathrm{P}<0.05)$. Data were taken at $7^{\text {th }}$ day of treatments.

\begin{tabular}{|c|c|c|c|}
\hline \multirow{2}{*}{ Genotpes } & \multicolumn{3}{|c|}{ Treatments } \\
\hline & Control & $500 \mu \mathrm{M}$ & $1 \mathrm{mM}$ \\
\hline Kheri & $46.9667 \pm 3.2331^{\mathrm{b}}$ & $41.8667 \pm 4.9440^{\mathrm{ab}}$ & $37.8667 \pm 3.3005^{\mathrm{a}}$ \\
\hline Kalyansona & $45.1333 \pm 2.6388^{\mathrm{a}}$ & $43.3333 \pm 1.1590^{\mathrm{a}}$ & $39.8000 \pm 4.7286^{\mathrm{a}}$ \\
\hline Sonora-64 & $43.4667 \pm 3.5852^{\mathrm{b}}$ & $45.6333 \pm 3.0139^{b}$ & $33.0667 \pm 2.4786^{\mathrm{a}}$ \\
\hline Sonalika & $42.4667 \pm 3.6501^{\mathrm{a}}$ & $41.5667 \pm 3.3546^{\mathrm{a}}$ & $42.2000 \pm 2.0952^{\mathrm{a}}$ \\
\hline Pavon-76 & $48.7667 \pm 1.6921^{a}$ & $48.1667 \pm 2.6006^{\mathrm{a}}$ & $48.9333 \pm 6.9515^{\mathrm{a}}$ \\
\hline Kanchan & $48.2667 \pm 1.7039^{\mathrm{a}}$ & $48.2000 \pm 0.9540^{\mathrm{a}}$ & $47.5333 \pm 1.7098^{\mathrm{a}}$ \\
\hline Sourav & $45.2667 \pm 4.2442^{\mathrm{a}}$ & $47.9000 \pm 2.4980^{\mathrm{a}}$ & $45.6667 \pm 3.2021^{\mathrm{a}}$ \\
\hline Gourab & $48.5667 \pm 1.0116^{a}$ & $42.0667 \pm 6.7988^{a}$ & $43.0667 \pm 5.1675^{a}$ \\
\hline Shatabdi & $45.7667 \pm 1.8903^{\mathrm{a}}$ & $44.1667 \pm 1.7388^{a}$ & $46.5000 \pm 1.3748^{\mathrm{a}}$ \\
\hline Sufi & $44.3667 \pm 2.8746^{\mathrm{a}}$ & $44.3667 \pm 3.2254^{\mathrm{a}}$ & $44.8667 \pm 0.6429^{\mathrm{a}}$ \\
\hline Bijoy & $44.7000 \pm 2.9206^{\mathrm{a}}$ & $44.4667 \pm 1.3317^{\mathrm{a}}$ & $44.2000 \pm 1.0440^{\mathrm{a}}$ \\
\hline Prodip & $44.2333 \pm 2.3116^{\mathrm{a}}$ & $43.5333 \pm 2.9771^{\mathrm{a}}$ & $41.1333 \pm 3.7687^{\mathrm{a}}$ \\
\hline BARI Gom-25 & $47.5333 \pm 0.5033^{\mathrm{a}}$ & $47.3333 \pm 2.5482^{\mathrm{a}}$ & $48.1333 \pm 4.0104^{\mathrm{a}}$ \\
\hline BARI Gom-29 & $40.3000 \pm 2.6058^{\mathrm{a}}$ & $39.9667 \pm 2.2591^{\mathrm{a}}$ & $37.1667 \pm 3.9552^{\mathrm{a}}$ \\
\hline BARI Gom-31 & $41.7333 \pm 0.9504^{b}$ & $38.2667 \pm 4.1053^{b}$ & $30.3333 \pm 4.1016^{a}$ \\
\hline BARI Gom-32 & $45.6667 \pm 2.2030^{\mathrm{b}}$ & $42.3333 \pm 3.3382^{\mathrm{ab}}$ & $40.0667 \pm 1.6653^{\mathrm{a}}$ \\
\hline BARI Gom-33 & $49.6667 \pm 1.5011^{\mathrm{a}}$ & $42.9000 \pm 4.4306^{\mathrm{a}}$ & $40.9667 \pm 9.1571^{\mathrm{a}}$ \\
\hline LalGom & $45.4000 \pm 2.1378^{\mathrm{a}}$ & $41.7000 \pm 6.6776^{\mathrm{a}}$ & $42.6000 \pm 3.2187^{\mathrm{a}}$ \\
\hline KhudeGom & $37.6667 \pm 4.5709^{\mathrm{b}}$ & $37.2333 \pm 1.9088^{b}$ & $30.9333 \pm 1.5948^{\mathrm{a}}$ \\
\hline Balaka & $45.6333 \pm 3.2332^{\mathrm{a}}$ & $44.0667 \pm 3.2517^{\mathrm{a}}$ & $40.1333 \pm 1.7616^{\mathrm{a}}$ \\
\hline Akbar & $42.8333 \pm 2.2942^{a}$ & $45.0000 \pm 2.1378^{a}$ & $43.5000 \pm 2.2113^{\mathrm{a}}$ \\
\hline Barkat & $48.9667 \pm 0.2887^{b}$ & $46.0333 \pm 1.3458^{a}$ & $44.8667 \pm 1.4295^{a}$ \\
\hline BARI Gom-26 & $43.4000 \pm 4.8867^{\mathrm{a}}$ & $42.5000 \pm 3.4828^{\mathrm{a}}$ & $36.6667 \pm 1.8448^{\mathrm{a}}$ \\
\hline BARI Gom-27 & $44.2667 \pm 1.3051^{\mathrm{a}}$ & $43.1000 \pm 5.2460^{\mathrm{a}}$ & $40.9667 \pm 4.5797^{\mathrm{a}}$ \\
\hline BARI Gom-28 & $43.2000 \pm 2.5515^{\mathrm{a}}$ & $43.1000 \pm 2.8054^{\mathrm{a}}$ & $40.5333 \pm 0.6028^{\mathrm{a}}$ \\
\hline BARI Gom-30 & $42.1000 \pm 2.9597^{\mathrm{b}}$ & $40.2333 \pm 4.8686^{\mathrm{b}}$ & $28.6333 \pm 5.4638^{\mathrm{a}}$ \\
\hline
\end{tabular}

sum indicates the most susceptible genotypes. The most tolerant (T) genotype was Akbar, despite all of the characters resulting in a minimal rank-sum (2.97), followed by BARI Gom-31 (3.45), Barkat (3.54) and Sufi (3.65), suggesting that these are more $\mathrm{Pb}$ tolerant (T) among the genotypes tested, while BARI Gom-26 (10.14), KhudeGom (9.69), BARI Gom-30 (8.79), LalGom (8.76) and BARI Gom-32 were the most sensitive (S) respectively. More scores were seen in the remaining genotypes, which we rated as moderately $\mathrm{Pb}$ tolerant (MT).

\section{Discussion}

Lead tolerance was the primary criteria for assessing genotypes in our study. The metal resistance contrast between species within the same genus is a significant method in assessing whether a physiological parameter is correlated with the tolerance of metals (40). In addition, the performance of phytoextraction is directly associated to the plant's ability to withstand and absorb contaminants in its over-ground parts $(18,27)$. In phytoremediation processes, suitable plant assortment is also an important factor (41). It was reported that hydroponic screening is a fast method of identifying of potential species of phytoremediation as an alternate option to expensive field studies (42). During our study, wheat plants display significant variation in growth characteristics, such as RL, SH, RDW, SDW and chlorophyll content under hydroponics conditions with applied $\mathrm{Pb}$ stress (Table 1-6).

Many research confirmed that the effect of the higher concentrations of phytotoxic metals on plant tissues was more hazardous than the effect of the lower concentrations by inhibiting the development of roots and aerial plant components (16, 43-44). According to the studies reported (45, (46), low $\mathrm{Pb}$ concentrations can stimulate plant development, but plant growth can be prevented by concentrations above $0.5 \mu \mathrm{M}$. The harmful effect of $\mathrm{Pb}$ on plant growth is time and dosage dependent in most instances (47-49). Our findings similarly showed differential variations in $\mathrm{Pb}$ tolerance in responses to different doses of $\mathrm{Pb}$ in wheat. In this study, genotypes displayed reduced growth parameters by $\mathrm{Pb}$ concentrations (500 $\mu \mathrm{M}$ and $1 \mathrm{mM}$ ) compared to the control. Similar $\mathrm{Pb}$ toxicity symptoms were seen in earlier research $(34,42,50-56)$. The toxic effect of $\mathrm{Pb}$ may be caused by affecting a number of physiological and biochemical systems, including ion toxicity, enzyme activity, respiration and photosynthesis $(57,58)$.

From our results, distinctly visible symptoms were observed in both root length and shoot height of wheat genotypes, but root growth was highly restricted by $\mathrm{Pb}$ (Fig. 1, Table 2), suggesting an increased number of secondary roots per unit root length, which were similar as of earlier findings (44). Roots are the first organ to come to $\mathrm{Pb}$ contact, providing the main path for metal ion penetration $(37,59)$. However, most of the absorbed $\mathrm{Pb}$ (about $95 \%$ or greater) are stored or uptake is restricted to roots, only a few are passed to aerial plant component (30-32), which is a good indication for tolerance. Upon entering the root, lead passes predominantly through apoplast and bind the sources of water before it enters the endodermis (30, 60 ). Balanced nutrients supply is vital for normal growth and development of the plant, while plants 
Table 7. The Ranking of various genotypes of wheat owing to the $\mathrm{Pb}$ stress. Compared to controls based on morphological criteria, the numerical number shows the tolerance rating of genotypes complemented by all $\mathrm{Pb}$ concentrations (RL=Root length, SH=Shoot height, RDW=Root dry weight, SDW=Shoot dry weight and SPAD=Total chlorophyll). Here, RS= Rank sum; $\overline{\mathbf{R}}=$ Rank mean; SDR=Standard deviation of rank.

\begin{tabular}{|c|c|c|c|c|c|c|c|c|c|c|}
\hline \multirow{2}{*}{ Genotypes } & \multicolumn{5}{|c|}{ Morphological Parameters } & \multirow{2}{*}{$\overline{\mathbf{R}}$} & \multirow{2}{*}{ SDR } & \multirow{2}{*}{ RS } & \multirow{2}{*}{$\begin{array}{c}\text { Genotype } \\
\text { Ranking }\end{array}$} & \multirow{2}{*}{$\begin{array}{c}\text { Tolerance } \\
\text { Degree }\end{array}$} \\
\hline & $\mathbf{R L}$ & SH & RDW & SDW & SPAD & & & & & \\
\hline Kheri & 5.5 & 9 & 1.5 & 1 & 1.5 & 3.7 & 3.475 & 7.17 & 17 & MT \\
\hline Kalyansona & 5 & 8 & 6.5 & 1 & 1.5 & 4.4 & 3.070 & 7.47 & 19 & MT \\
\hline Sonora-64 & 4 & 8 & 6 & 1 & 1.5 & 4.1 & 2.966 & 7.07 & 15 & MT \\
\hline Sonalika & 6 & 8.5 & 6 & 1 & 1.5 & 4.6 & 3.229 & 7.83 & 20 & MT \\
\hline$\overline{\text { Pavon-76 }}$ & 1.5 & 4.5 & 6 & 1 & 1.5 & 2.9 & 2.219 & 5.12 & 11 & MT \\
\hline Kanchan & 2 & 1 & 5.5 & 1 & 1.5 & 2.2 & 1.891 & 4.09 & 5 & MT \\
\hline Sourav & 3 & 5 & 5 & 1 & 1 & 3.0 & 2.000 & 5.00 & 10 & MT \\
\hline Gourab & 4 & 3 & 4.5 & 1 & 1.5 & 2.8 & 1.525 & 4.32 & 6 & MT \\
\hline Shatabdi & 4 & 6 & 3.5 & 1 & 1 & 3.1 & 2.133 & 5.23 & 12 & MT \\
\hline$\overline{\text { Sufi }}$ & 5 & 1 & 1 & 1 & 1.5 & 1.9 & 1.746 & 3.65 & 4 & $\mathrm{~T}$ \\
\hline Bijoy & 4 & 4.5 & 4.5 & 1 & 1 & 3.0 & 1.837 & 4.84 & 8 & MT \\
\hline Prodip & 5.5 & 2.5 & 2.5 & 1 & 1 & 2.5 & 1.837 & 4.34 & 7 & MT \\
\hline BARI Gom-25 & 1 & 7 & 3.5 & 1 & 1 & 2.7 & 2.636 & 5.34 & 13 & MT \\
\hline BARI Gom-29 & 4 & 3.5 & 5.5 & 1 & 1.5 & 3.1 & 1.851 & 4.95 & 9 & MT \\
\hline BARI Gom-31 & 3.5 & 2 & 3.5 & 1 & 1.5 & 2.3 & 1.151 & 3.45 & 2 & $\mathrm{~T}$ \\
\hline BARI Gom-32 & 5 & 9 & 7.5 & 1 & 1.5 & 4.8 & 3.546 & 8.35 & 22 & $\mathrm{~S}$ \\
\hline BARI Gom-33 & 4.5 & 8.5 & 5.5 & 1 & 1.5 & 4.2 & 3.074 & 7.27 & 18 & MT \\
\hline LalGom & 5 & 9 & 8.5 & 1 & 1.5 & 5.0 & 3.758 & 8.76 & 23 & $\mathrm{~S}$ \\
\hline KhudeGom & 7 & 10 & 8.5 & 1 & 2 & 5.7 & 3.994 & 9.69 & 25 & $\mathrm{~S}$ \\
\hline Balaka & 4 & 7 & 6 & 1 & 1.5 & 3.9 & 2.655 & 6.56 & 14 & MT \\
\hline Akbar & 3 & 2.5 & 2.5 & 1 & 1 & 2.0 & 0.935 & 2.94 & 1 & $\mathrm{~T}$ \\
\hline Barkat & 2.5 & 1 & 4.5 & 1 & 1 & 2.0 & 1.541 & 3.54 & 3 & $\mathrm{~T}$ \\
\hline BARI Gom-26 & 6 & 11 & 9 & 1 & 1.5 & 5.7 & 4.438 & 10.14 & 26 & $\mathrm{~S}$ \\
\hline BARI Gom-27 & 5 & 8 & 5.5 & 1 & 1.5 & 4.2 & 2.928 & 7.13 & 16 & MT \\
\hline BARI Gom-28 & 5.5 & 9 & 6.5 & 1 & 1.5 & 4.7 & 3.402 & 8.10 & 21 & MT \\
\hline BARI Gom-30 & 7 & 10 & 5.5 & 1 & 1.5 & 5.0 & 3.791 & 8.79 & 24 & $\mathrm{~S}$ \\
\hline LSD value & 1.337 & 1.302 & 0.009 & 0.009 & 3.88 & - & - & - & - & - \\
\hline
\end{tabular}

have limited nutrient content in Pb stress. That can be attributed to the physical disruption of roots absorption sites that cannot absorb several ions (61).

For dry weight of plant parts, considerable reductions were observed under $\mathrm{Pb}$ treatment. Similar phenomena were also described in wheat and lentils (62), in Pisum sativum (63), in Plantago major (64), in Zea mays (65). Similarly, in tomato seedlings, fresh and dry biomass of roots, shoots and leaves were negatively affected by increasing $\mathrm{Pb}$ concentrations (66). These symptoms can be essentially attributed to a deficiency of macroelements.

The core components of photosynthesis and biomass production in plants are chloroplast pigments, Chlorophyll. In the wheat seedlings in our study, Chl contents were already significantly lowered at $500 \mu \mathrm{M}$ $\mathrm{Pb}$ and this effect was even more pronounced at $1 \mathrm{mM}$ $\mathrm{Pb}$ compared with the control (Table 6). Nevertheless, $\mathrm{Pb}$-treated plants displayed decreased biosynthesis of $\mathrm{Chl}$ content and heightened deterioration of $\mathrm{Chl}$ due to increased chlorophyllase activity (67), the disturbance of the amount of chloroplastic stroma triggered by ROS $(58,68)$ or a lack of availability of nutrients such as $\mathrm{Mg}$ and Fe (69). From the results in this study, high Chl score findings suggest a lower chlorophyll synthesis disruption and hence a higher lead resistance from this parameter. In addition, for screening several species, Chl has been used as a parameter in corn (70), in wheat (71), in sweet pepper (72) and in field peas (73).
Wheat genotypes exhibited significant variation in all growth parameters, indicating that these parameters could be used as a selection criterion for $\mathrm{Pb}$ tolerance in the hydroponic conditions. Noticeably, it is argumentative to recognize the $\mathrm{Pb}$ tolerant genotypes based on a single criterion. The results of our study are comforted with earlier works (74-77). Also, similar ranking procedure was followed in screening rapeseed and mustard genotypes for salt tolerance (78), in Brassica varieties (79) for drought tolerance and in rice (80) for cadmium tolerance. Previously it was proved that the morphological score is well correlated to the physiological state of plants and used as rapid screening for stress tolerance (80). In all parameters, Akbar consistently displayed superior tolerance, followed by other genotypes. This genotype can be used in future breeding programmes to develop lead-tolerant wheat cultivars. We also suggest that farmers cultivate Akbar in $\mathrm{Pb}$ polluted soils in order to ensure improved crop yield relative to other varieties evaluated.

\section{Conclusion}

This study concluded that $\mathrm{Pb}$ stress may have severe effects on wheat yield and quality characters by changing its morpho-physiological traits and chlorophyll content and noteworthy genotypic differences were found. Among studied wheat genotypes Akbar proved tolerant to lead $(\mathrm{Pb})$, 


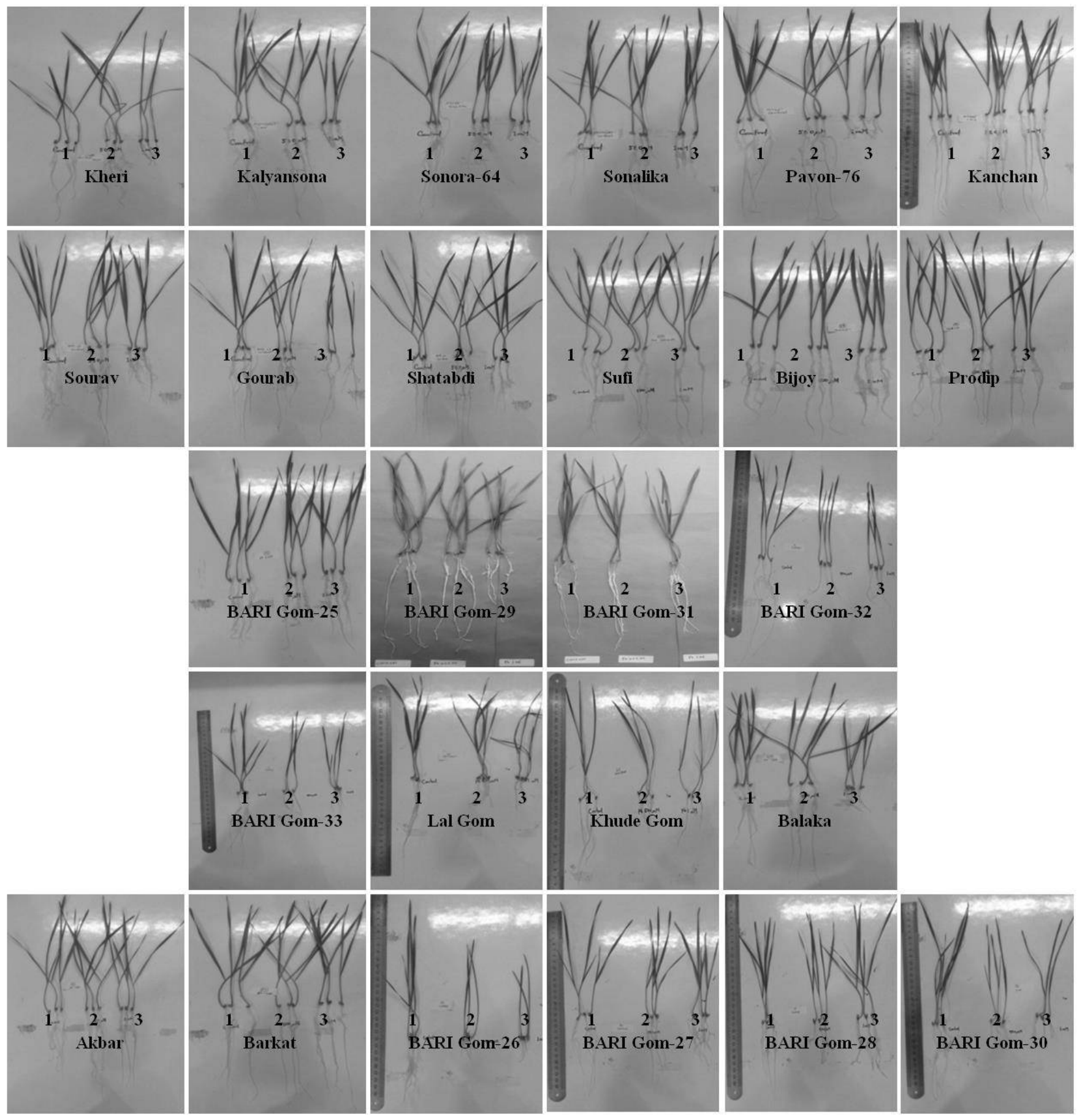

Fig.1. Phenotypes of different wheat genotypes grown hydroponically with or without $\mathrm{Pb}$ stress at $7^{\text {th }}$ day of treatments. Numeric letters 1 , 2 and 3 indicate control, $500 \mu \mathrm{M} \mathrm{Pb}\left(\mathrm{NO}_{3}\right)_{2}$ and $1 \mathrm{mM} \mathrm{Pb}\left(\mathrm{NO}_{3}\right)_{2}$ respectively.

suggesting this genotype is prominent resource and could be used as genetic materials for the further breeding programme.

\section{Acknowledgements}

Authors acknowledge the financial support of Rajshahi University (grant no. A-5/52/RU/Bio-20/20192020) for conduction this research work.

\section{Authors' contributions}

MMR performed most of the experiments and prepared the draft manuscript. MRAM conducted data measurement. AHK and MFA supervised the whole work.

\section{Conflict of interests}

Authors declare they have no conflict of interest.

\section{References}

1. Skovmand B, Reynolds MP. Increasing yield potential for marginal areas by exploring genetic resources collection. The eleventh regional wheat workshop for Eastern, Central and Southern Africa, Addis Ababa, Ehiopia; 2000.18(22): 67-77.

2. Ortiz R, Sayre KD, Govaerts B, Gupta R, Subbarao GV, Ban T, Hodson D, Dixon JM, Ortiz-Monasterio JI, Reynolds M. Climate 
change: Can wheat beat the heat? Agriculture, Ecosystems and Environment. https://doi.org/10.1016/j.agee.2008.01.019

3. Singh K, Sharma SN, Sharma Y. Effect of high temperature on yield attributing traits in bread wheat. Bangladesh Journal of Agricultural Research. https://doi.org/10.3329/bjar.v36i3.9270

4. Athar HR, Khan A, Ashraf M. Exogenously applied ascorbic acid alleviates salt-induced oxidative stress in wheat Environmental and Experimental Botany. 2008.63(1-3):224-31. https://doi.org/10.1016/j.envexpbot.2007.10.018

5. Rahaie M, Xue GP, Schenk PM. The role of transcription factors in wheat under different abiotic stresses. Abiotic Stress-Plan Responses and Applications in Agriculture. Kourosh Vahdati K, Leslie C (eds.). Rijeka, Croatia: InTech; 2013. p. 367-85. http://dx.doi.org/10.5772/54795

6. Rodriguez M, Canaless E, Borrás-Hidalgo O. Molecular aspects of abiotic stress in plants. Biotecnología Aplicada. 2005;22 (1):110.

http://www.esalq.usp.br/lepse/imgs/conteudo_thumb/Molecula r-Aspects-of-Abiotic-Stress-in-Plants.pdf

7. Rodríguez-Eugenio N, McLaughlin M, Pennock D. Soil Pollution: a hidden reality. Rome, FAO; 2018. p. 142.

8. Shahid M, Dumat C, Pourrut B, Abbas G, Shahid N, Pinelli E. Role of metal speciation in lead-induced oxidative stress to Vicia faba roots. Russian Journal of Plant Physiology. 2015;62(4):448-54. https://doi.org/10.7868/S0015330315040156

9. Khalid S, Shahid M, Niazi NK, Rafiq M, Bakhat HF, Imran M, Abbas T, Bibi I, Dumat C. Arsenic behaviour in soil-plant system: Biogeochemical reactions and chemical speciation influences. Enhancing Cleanup of Environmental Pollutants, Springer: Berlin, Germany; 2017. p. 97-140. http://dx.doi.org/10.1007/978-3-319-55423-5_4

10. Gratão PL, Polle A, Lea PJ, Azevedo RA. Making the life of heavy metal-stressed plants a little easier. Functional Plant Biology. 2005;32(6):481-94. https://doi.org/10.1071/FP05016

11. Merian E. Introduction on environmental chemistry and global cycles of chromium, nickel, cobalt beryllium, arsenic, cadmium and selenium and their derivatives. Toxicological and Environmental Chemistry. 1984;8(1):9-38. https://doi.org/10.1080/02772248409357038

12. Khan FU, Rahman AU, Jan A, Riaz M. Toxic and trace metals ( $\mathrm{Pb}, \mathrm{Cd}, \mathrm{Zn}, \mathrm{Cu}, \mathrm{Mn}, \mathrm{Ni}, \mathrm{Co}$ and $\mathrm{Cr}$ ) in dust, dustfall/soil. Journal of the Chemical Society of Pakistan. 2004;26(4):453-56.

13. Pourrut B, Shahid M, Dumat C, Winterton P, Pinelli E. Lead uptake, toxicity and detoxification in plants. Reviews of Environmental Contamination and Toxicology. 2011;213:11336. https://doi.org/10.1007/978-1-4419-9860-6_4

14. Li X, Wai OWH, Li YS, Coles BJ, Ramsey MH, Thornton I. Heavy metal distribution in sediment profiles of the Pearl River estuary, South China. Applied Geochemistry. 2000; 15(5):56781. https://doi.org/10.1016/S0883-2927(99)00072-4

15. Seaward MRD, Richardson DHS. Atmospheric sources of metal pollution and effects on vegetation. In: Shaw AJ (ed) Heavy metal tolerance in plants: Evolutionary Aspects. CRC Press Inc, Boca Raton, FL; 1990. p. 75-92.

16. Islam E, Yang X, Li T, Liu D, Jin X, Meng F. Effect of Pb toxicity on root morphology, physiology and ultrastructure in the two ecotypes of Elsholtzia argyi. Journal of Hazardous Materials. 2007;147(3):806-16.

https://doi.org/10.1016/j.jhazmat.2007.01.117

17. Freitas EV, Nascimento CW, Souza A, Silva FB. Citric acidassisted phytoextraction of lead: A field experiment. Chemosphere. https://doi.org/10.1016/j.chemosphere.2013.01.103

18. Huang JW, Chen J, Berti WR, Cunningham SD. Phytoremediation of lead-contaminated soils: Role of synthetic chelates in lead phytoextraction. Environmental Science and Technology. https://doi.org/10.1021/es9604828 1997;31(3):800-05.

19. Salazar MJ, Pignata ML. Lead accumulation in plants grown in polluted soils. Screening of native species for phytoremediation. Journal of Geochemical Exploration 2014;137:29-36. https://doi.org/10.1016/j.gexplo.2013.11.003

20. Uzu G, Sobanska S, Aliouane Y, Pradere P, Dumat C. Study of lead phytoavailability for atmospheric industrial micronic and sub-micronic particles in relation with lead speciation Environmental Pollution. 2009;157(4):1178-85. https://doi.org/10.1016/j.envpol.2008.09.053

21. Tabelin CB, Igarashi T. Mechanisms of arsenic and lead release from hydrothermally altered rock. Journal of Hazardous Materials.

https://doi.org/10.1016/j.jhazmat.2009.04.049

22. Sammut ML, Noack Y, Rose J, Hazemann JL, Proux O, Depoux $\mathrm{M}$, Ziebel A, Fiani E. Speciation of $\mathrm{Cd}$ and $\mathrm{Pb}$ in dust emitted from sinter plant. Chemosphere. 2010;78(4):445-50. https://doi.org/10.1016/j.chemosphere.2009.10.039

23. Vega FA, Andrade ML, Covelo EF. Influence of soil properties on the sorption and retention of cadmium, copper and lead, separately and together, by 20 soil horizons: Comparison of linear regression and tree regression analyses. Journal of Hazardous Materials. 2010;174(1-3):522-33. https://doi.org/10.1016/j.jhazmat.2009.09.083

24. Hamby DM. Site remediation techniques supporting environmental restoration activities-a review. Science of The Total Environment. 1996;191(3):203-24. https://doi.org/10.1016/ S0048-9697(96)05264-3

25. Henry JR. An overview of the phytoremediation of lead and mercury. National Network of Environmental Management Studies (NNEMS) fellow. U.S. Environmental Protection Agency, Office of Solid Waste and Emergency Response, Technology Innovation Office, Washington, D.C; 2000.

26. Pulford ID, Watson C. Phytoremediation of heavy metalcontaminated land by trees - a review. Environment International. https://doi.org/10.1016/S0160-4120(02)00152-6

27. Lonardo DS, Capuana M, Arnetoli M, Gabbrielli R, Gonnelli C. Exploring the metal phytoremediation potential of three Populus alba L. clones using an in vitro screening. Environmental Science and Pollution Research. 2011;18(1):82 90. https://doi.org/10.1007/s11356-010-0354-7

28. Bhargava A, Carmona FF, Bhargava M, Srivastava S Approaches for enhanced phytoextraction of heavy metals. Journal of Environmental Management. 2012;105:103-20. https://doi.org/10.1016/j.jenvman.2012.04.002

29. Gong Y, Zhao D, Wang Q. An overview of field-scale studies on remediation of soil contaminated with heavy metals and metalloids: Technical progress over the last decade. Water Research.

https://doi.org/10.1016/j.watres.2018.10.024

2018;147:440-60.

30. Lane SD, Martin ES. A histochemical investigation of lead uptake in Raphanus sativus. The New Phytologist. 1977; 79(2):281-86. https://doi.org/10.1111/j.1469-8137.1977.tb02206.x

31. Woźny A, Schneider J, E. A. Gwóźdź EA. The effects of lead and kinetin on greening of barley leaves. Biologia Plantarum. 1995;37:541-52. https://doi.org/10.1007/BF02908836

32. Kumar PBAN, Dushenkov V, Motto H, Raskin I Phytoextraction: The use of plants to remove heavy metals from soils. Environmental Science and Technology. 1995;29(5):1232-38. https://doi.org/10.1021/es00005a014

33. Purdy JJ, Smart LB. Hydroponic screening of shrub willow (Salix spp.) for arsenic tolerance and uptake. International Journal of Phytoremediation. 2008;10(6):515-28. https://doi.org/ $10.1080 / 15226510802115000$

34. Zhivotovsky OP, Kuzovkina JA, Schulthess CP, Morris T, Pettinelli D, Ge M. Hydroponic screening of willows (Salix L.) for lead tolerance and accumulation. International Journal of Phytoremediation. https://doi.org/10.1080/15226511003671361

35. Watson C, Pulford ID, Riddell-Black D. Screening of willow species for resistance to heavy metals: Comparison of performance in a hydroponics system and field trials. International Journal of Phytoremediation. 2003;5(4):351-65. https://doi.org/10.1080/15226510309359042 
36. Hoagland DR, Arnon DI. The water-culture method for growing plants without soil. Circular. California Agricultural Experiment Station. 1950;347(2):1-32.

37. Kaur G, Singh HP, Batish DR, Kohli RK. Lead (Pb)-induced biochemical and ultrastructural changes in wheat (Triticum aestivum) roots. Protoplasma. 2013;250(1):53-62. https://doi.org/10.1007/s00709-011-0372-4

38. Peng S, Garcia FV, Laza RC, Cassman KG. Adjustment for specific leaf weight improves chlorophyll meter's estimate of rice leaf nitrogen concentration. Agronomy Journal. 1993;85:987-90.

https://doi.org/10.2134/agronj1993.00021962008500050005x

39. Farshadfar E, Elyasi P, Aghaee M. In vitro selection for drought tolerance in common wheat (Triticum aestivum L.) genotypes by mature embryo culture. American Journal of Scientific Research. 2012a;48:102-15.

40. K“ohl KI, L“osch R. Experimental characterization of heavy metal tolerance in plants. In: Prasad MNV, Hagemeyer J (eds.) Heavy Metal Stress in Plants. Berlin (Germany): Springer; 1999. p. 371-389. https://link.springer.com/chapter/10.1007/978-3662-07745-0 17

41. Angelova V, Ivanova R, Todorov G, Ivanov K. Heavy metal uptake by Rape. Communications in Soil Science and Plant Analysis.

https://doi.org/10.1080/00103620701826480

42. Huang JW, Cunningham SD. Lead phytoextraction: Species variation in lead uptake and translocation. The New Phytologist. 1996;134(1):75-84. https://doi.org/10.1111/j.14698137.1996.tb01147.x

43. Zhi-xin N, Li-na S, Tie-heng S, Yu-shuang L, Hong W. Evaluation of phytoextracting cadmium and lead by sunflower, ricinus, alfalfa and mustard in hydroponic culture. Journal of Environmental Sciences. 2007;19(8):961-67. https://doi.org/10.1016/S1001-0742(07)60158-2

44. Kopittke PM, Asher CJ, Kopittke RA, Menzies NW. Toxic effects of $\mathrm{Pb}^{2+}$ on growth of cowpea (Vigna unguiculata). $\begin{array}{lll}\text { Environmental } & \text { Pollution. }\end{array}$ https://doi.org/10.1016/j.envpol.2007.01.011

45. Patra J, Lenka M, Panda BB. Tolerance and co-tolerance of the grass Chloris barbata Sw. to mercury, cadmium and zinc. The New Phytologist. 1994;128(1):165-71. https://doi.org/10.1111/j.1469-8137.1994.tb03999.x

46. Seregin IV, Ivanov VB. Physiological aspects of cadmium and lead toxic effects on higher plants. Russian Journal of Plant Physiology. http://dx.doi.org/10.1023/A:1016719901147

47. Dey SK, Dey J, Patra S, Pothal D. Changes in the antioxidative enzyme activities and lipid peroxidation in wheat seedlings exposed to cadmium and lead stress. Brazilian Journal of Plant Physiology. 2007;19(1):53-60. https://doi.org/10.1590/S167704202007000100006

48. Gupta DK, Nicoloso FT, Schetinger MRC, Rossato LV, Pereira LB, Castro GY, Srivastava S, Tripathi RD. Antioxidant defense mechanism in hydroponically grown Zea mays seedlings under moderate lead stress. Journal of Hazardous Materials. 2009;172(1):479-84.

https://doi.org/10.1016/j.jhazmat.2009.06.141

49. Gupta DK, Huang H, Yang XE, Razafindrabe BHN, Inouhe M. The detoxification of lead in Sedum alfredii $\mathrm{H}$. is not related to phytochelatins but the glutathione. Journal of Hazardous Materials. https://doi.org/10.1016/j.jhazmat.2009.12.052

50. Burton KW, Morgan E, Roig A. The influence of heavy metals upon the growth of sitka-spruce in South Wales forests. II. Greenhouse experiments. Plant and Soil. 1984;78(3):271-82. https://www.jstor.org/stable/42934580

51. Påhlsson, AMB. Toxicity of heavy metals $(\mathrm{Zn}, \mathrm{Cu}, \mathrm{Cd}, \mathrm{Pb})$ to vascular plants: A literature review. Water, air and soil Pollution.

https://doi.org/10.1007/BF00279329

52. Verma S, Dubey RS. Lead toxicity induces lipid peroxidation and alters the activities of antioxidant enzymes in growing rice $\begin{array}{lll}\text { plants. } & \text { Plant 2003;164(4):645-55. }\end{array}$ https://doi.org/10.1016/S0168-9452(03)00022-0
53. Sharma P, Dubey RS. Lead toxicity in plants. Brazilian Journal of Plant Physiology. 2005;17(1):35-52. https://doi.org/10.1590/S1677-04202005000100004

54. Mun HW, Hoe AL, Koo LD. Assessment of $\mathrm{Pb}$ uptake, translocation and immobilization in kenaf (Hibiscus cannabinus L.) for phytoremediation of sand tailings. Journal of Environmental Sciences. 2008;20(1):1341-47. https://doi.org/10.1016/s1001-0742(08)62231-7

55. Liu W, Zhou Q, Zhang Y, Wei S. Lead accumulation in different Chinese cabbage cultivars and screening for pollution-safe cultivars. Journal of Environmental Management. 2010;91:78188. https://doi.org/10.1016/j.jenvman.2009.10.009

56. Rezvani M, Zaefarian F. Bioaccumulation and translocation factors of cadmium and lead in Aeluropus littoralis. Australian Journal of Agricultural Engineering. 2011;2(4):114-19. http://sciencej.com/rezvani_2_4_2011_114_119.pdf

57. Stevens RG, Creissen GP, Mullineaux PM. Clonning and characterization of a cytosolic glutathione reductase cDNA from pea (Pisum sativum L.) and its expression in response to stress. Plant Molecular Biology. 1997;35:641-54. https://doi.org/ 10.1023/a:1005858120232

58. Hadi F, Aziz T. A mini review on lead $(\mathrm{Pb})$ toxicity in plants. Journal of Biology and Life Science. 2015;6(2):91-101. https://doi.org/10.5296/jbls.v6i2.7152

59. Piechalak A, Tomaszewska B, Baralkiewicz D, Malecka A. Accumulation and detoxification of lead ions in legumes. Phytochemistry.

https://doi.org/10.1016/S0031-9422(02)00067-5

60. Tanton TW, Crowdy SH. The distribution of lead chelate in the transpiration stream of higher plants. Pesticide Science. 1971;2(5):211-13. https://doi.org/10.1002/ps.2780020507

61. Lamhamdi M, Galiou OE, Bakrim A, Nóvoa-Muñoz JC, AriasEstévez M, Aarab A, Lafont R. Effect of lead stress on mineral content and growth of wheat (Triticum aestivum) and spinach (Spinacia oleracea) seedlings. Saudi Journal of Biological Sciences. https://doi.org/10.1016/j.sjbs.2012.09.001

62. Mesmar MN, Jaber K. The toxic effect of lead on seed germination, growth, chlorophyll and protein contents of wheat and lens. Acta Biologica Hungarica. 1991;42(4):331-44. PMID: 1841484

63. Kevresan S, Petrovic N, Popovic M, Kandrac J. Nitrogen and protein metabolism in young pea plants as affected by different concentrations of nickel, cadmium, lead and molybdenum. Journal of Plant Nutrition. 2001;24(10):1533 1644. https://doi.org/10.1081/PLN-100106026

64. Kosobrukhov A, Knyazeva I, Mudrik V. Plantago major plants responses to increase content of lead in soil: Growth and photosynthesis. Plant Growth Regulation. 2004;42:145-51. https://doi.org/10.1023/B\%3AGROW.0000017490.59607.6b

65. Çimrin KM, Turan M, Kapur B. Effect of elemental sulphur on heavy metals solubility and remediation by plants in calcareous soils. Fresenius Environmental Bulletin. 2007;16(9):1113-20. HERO ID: 461243

66. Akinci IE, Akinci S, Yilmaz K. Response of tomato (Solanum lycopersicum L.) to lead toxicity: Growth, element uptake, chlorophyll and water content. African Journal of Agricultura Research. 2010;5(6):416-23. https://dx.doi.org/10.5897/AJAR10

67. Drazkiewicz M. Chlorophyllase: occurrence, functions, mechanism of action, effects of external and internal factors Photosynthetica. 1994;30:321-31. https://doi.org/10.1093/jxb/42.1.1

68. Stefanov K, Seizova K, Popova I, Petkov V, Kimenov G, Popov S. Effects of lead ions on the phospholipids composition in leaves of Zea mays and Phaseolus vulgaris. Journal of Plant Physiology. 1995;147(2):243-46. https://doi.org/10.1016/S01761617(11)81511-7

69. Burzynski M. Influence of lead and cadmium on the absorption and distribution of potassium, calcium, magnesium and iron in cucumber seedlings. Acta Physiologiae Plantarum. 1987;9:229-38. Corpus ID: 100825660

70. Dwyer LM, Tollenaar M, Houwing L. A non-destructive method to monitor leaf greenness in corn. Canadian Journal of Plant Science. 1991;71:505-09. https://doi.org/10.4141/cjps91-070 
71. Reeves DW, Mask PL, Wood CW, Delano DP. Determination of wheat nitrogen status with hand-helo chlorophyll meter: Influence of management practices. Journal of Plant Nutrition. 1993;16(5):781-89. https://doi.org/10.1080/01904169309364574

72. Madeira AC, Ferreira A, de Varennes A, Vieira MI. SPAD meter versus tristimulus colorimeter to estimate chlorophyll content and leaf color in sweet pepper. Communications in Soil Science and Plant Analysis. 2003;34(17-18):2461-70. https://doi.org/10.1081/CSS-120024779

73. Yakop UM. Physiological and genetic investigations of iron deficiency in field peas (Pisum sativum L.). PhD Thesis. School of Agriculture, Food and Wine.Faculty of Science. University of Adelaide, Waite Campus, PMB1, Glen Osmond, SA 5064 , Australia; 2012. http://hdl.handle.net/2440/78660

74. Karimizadeh R, Mohammadi M. Association of canopy temperature depression with yield of durum wheat genotypes under supplementary irrigation and rain-fed conditions. Australian Journal of Crop Science. 2011;5(2):138-46. http://www.cropj.com/karimizadeh_5_2_2011_138_146.pdf

75. Farshadfar E, Jamshidi B, Aghaee M. Biplot analysis of drought tolerance indicators in bread wheat lanraces of Iran. International Journal of Agriculture and Crop Sciences. 2012b;4(5):226-33. www.ijagcs.com IJACS/2012/4-5/226-233

76. Farshadfar E, Sheibanirad A, Soltanian M. Screening landraces of bread wheat genotypes for drought tolerance in the field and laboratory. International Journal of Farming and Allied Sciences. 2014;3(3):304-11.

77. Khalili M, Naghavi MR, Aboughadareh AP, Talebzadeh SJ. Evaluating of drought stress tolerance based on selection indices in spring canola cultivars (Brassica napus L.). Journal of Agricultural Science. 2012;4(11):78-85. https://doi.org/10.5539/jas.v4n11p78
78. Islam MR. Screening of rapeseed and mustard varieties of Bangladesh for salt tolerance.MS thesis, Dept. of Genetics and Plant Breeding, Bangladesh Agricultural University, Mymensingh, Bangladesh;1997.

79. Hossain MA, Robin ABMAHK, Hossain MA, Raffi SA. Screening of oilseed Brassica varieties for drought tolerance at the early stages of plant growth. Journal of the Bangladesh Agricultural University. http://dx.doi.org/10.22004/ag.econ.276579 2007;5(1):23-29.

80. Bari MA, Kabir AH, Reza MA. Rapid screening of cadmium tolerant rice (Oryza sativa L.) through morpho-physiologica markers. Plant Environment Development. 2018.7(2):1-9. http://plantenvdev.com/volume-7-number-2-july-2018-ped/

Additional information

Peer review information: Plant Science Today thanks Sectional Editor and the other anonymous reviewers for their contribution to the peer review of this work.

Reprints and permissions information is available at

https://horizonepublishing.com/journals/index.php/PST/open_access_policy

Publisher's Note: Horizon e-Publishing Group remains neutral with regard to jurisdictional claims in published maps and institutional affiliations.

To cite this article: Rahman M M R, Mintu M R A, Kabir A H, Alam M F. Genotypic screening of wheat and their physiological responses under lead toxicity. Plant Science Today. 2021;8(3):456-466. https://doi.org/10.14719/ pst.2021.8.3.1092

Plant Science Today, published by Horizon e-Publishing Group, is covered by Scopus, Web of Science, BIOSIS Previews, Clarivate Analytics, etc. See https://horizonepublishing.com/journals/index.php/PST/indexing_abstracting 